\title{
Excitonic optical nonlinearities and transport in the layered compound semiconductor
} GaSe

Mizeikis, V.; Vadim, Lyssenko; Østergaard, John Erland; Hvam, Jørn Märcher

Published in:

Physical Review B

Link to article, DOI:

10.1103/PhysRevB.51.16651

Publication date:

1995

Document Version

Publisher's PDF, also known as Version of record

Link back to DTU Orbit

Citation (APA):

Mizeikis, V., Vadim, L., Østergaard, J. E., \& Hvam, J. M. (1995). Excitonic optical nonlinearities and transport in the layered compound semiconductor GaSe. Physical Review B, 51(23), 16651-16659.

https://doi.org/10.1103/PhysRevB.51.16651

\section{General rights}

Copyright and moral rights for the publications made accessible in the public portal are retained by the authors and/or other copyright owners and it is a condition of accessing publications that users recognise and abide by the legal requirements associated with these rights.

- Users may download and print one copy of any publication from the public portal for the purpose of private study or research.

- You may not further distribute the material or use it for any profit-making activity or commercial gain

- You may freely distribute the URL identifying the publication in the public portal 


\title{
Excitonic optical nonlinearities and transport in the layered compound semiconductor GaSe
}

\author{
V. Mizeikis, ${ }^{*}$ V. G. Lyssenko, ${ }^{\dagger}$ J. Erland, ${ }^{\ddagger}$ and J. M. Hvam \\ Mikroelektronik Centret, The Technical University of Denmark, DK-2800 Lyngby, Denmark
}

(Received 27 December 1994)

\begin{abstract}
Dephasing and transient grating experiments in the direct excitonic absorption region of GaSe at low temperatures show that a fast relaxation within the one-dimensionally disordered excitonic band results in band filling being the dominant mechanism of the optical nonlinearity. Correspondingly, we observe a blueshift of the nonlinear signal with excitation density. The temperature dependence of the exciton diffusion constant measured in directions parallel to the GaSe layer planes indicates that temperatureindependent scattering (trapping) and scattering by acoustic phonons determine the exciton mobility at low temperatures.
\end{abstract}

\section{INTRODUCTION}

In the layered compound semiconductor GaSe, a high density of stacking faults between the layers cancels the translational symmetry along the crystallographic $c$ axis, while it is retained in the plane perpendicular to it. ${ }^{1}$ Consequently, features of both crystalline and disordered solid states are present in this material along different directions.

The most common consequences of disorder on the excitonic states are inhomogeneously broadened absorption lines and localization. To study these effects, nonlinear optical techniques like transient degenerate four-wave mixing (DFWM) have been successfully applied. Coherent dephasing and incoherent transient lightinduced grating experiments, performed on a variety of materials including bulk $\mathrm{CdS},{ }^{2} \mathrm{CdSe},{ }^{3}$ mixed crystals of $\mathrm{CdS}_{1-x} \mathrm{Se}_{x},{ }^{4}$ and molecular-beam epitaxy (MBE) grown $\mathrm{GaAs} / \mathrm{Al}_{x} \mathrm{Ga}_{1-x} \mathrm{As}$ multiple quantum wells ${ }^{5}$ have demonstrated various implications of disorder, anisotropy, and low-dimensional confinement.

Stacking fault disorder in GaSe produces inhomogeneous line shapes, described by a stochastic superposition of narrow homogeneously broadened lines. In most cases, the inhomogeneous line has a fine structure due to the predominance of some stacking fault sequences, and exciton cross-layer transport is inhibited by localization. Transport in directions along the layer planes is somewhat less investigated. An ideal crystalline structure of GaSe in these directions should result in fairly good transport properties, as evidenced by a carrier Hall mobility of $800 \mathrm{~cm}^{2} / \mathrm{V} \mathrm{s}$ at $80 \mathrm{~K} .{ }^{6}$ Surprisingly, transient grating studies at lower temperatures ${ }^{7,8}$ indicate no excitonic transport, suggesting very low exciton mobility or localization even in these directions. Lifetimes found from the transient grating decay are short $\left(T_{1} \approx 100 \mathrm{ps}\right)$ and attributed to recombination ${ }^{7}$ or scattering to the indirect valleys, ${ }^{8}$ while time-resolved luminescence ${ }^{9}$ and differential transmission experiments ${ }^{10}$ give longer, nanosecond exciton lifetimes.

The aim of this work is to investigate possible implications of disorder on exciton transport in directions paral- lel to the GaSe layers. For these studies we use the DFWM technique in the form of dephasing and transient light-induced grating experiments. Although dephasing experiments cannot monitor exciton transport, we apply it to investigate peculiarities of the nonlinear mechanism in GaSe, that are important for understanding the results obtained in the transient grating experiment. By spectrally resolving the DFWM signal, we detect effects of band filling in the one-dimensional exciton band. In the transient grating experiments, we determine the exciton diffusion constant and lifetime in the temperature range 7.5-65 K. Strong temperature-independent scattering and scattering by acoustic phonons are found to determine the exciton mobility at low temperatures.

\section{EXPERIMENTAL PROCEDURE}

Platelet samples of $\epsilon-\mathrm{GaSe}$ (thickness $\approx 15 \mu \mathrm{m}$ ), cleaved from single crystals, are used for the investigation. For light polarized perpendicularly to the $c$ axis, the $n=1$ direct free exciton line peaks at $2.1097 \mathrm{eV}$ (at helium temperature) and has a fine structure. The inhomogeneous width of the line is about $3 \mathrm{meV}$. Pulses from a dye laser (Rhodamine $6 \mathrm{G}$ ), synchronously pumped by a mode-locked argon-ion laser at a repetition rate of 82 $\mathrm{MHz}$ are used for excitation. By adjusting the dye laser cavity length, the temporal width of the generated pulses can be varied between 1- and 8-ps FWHM.

In the dephasing experiments two laser pulses with wave vectors $k_{1}$ and $k_{2}$ and frequency $\omega$, matching the energy of a resonance in the material, arrive at the sample with a mutual delay time $t_{12}$. The coherent polarization, induced by the first (pump) pulse, is detected after the time $t_{12}$ by the second (probe) pulse. The DFWM signal intensity $I_{\text {DFWM }}$ is recorded as a function of the delay time $t_{12}$ in the nearly phase-matched direction $2 \mathbf{k}_{2}-\mathbf{k}_{1}$. The decay is due to exciton scattering away from the coherent states, and in most cases can be described by the exponential law

$$
I_{\mathrm{DFWM}} \propto \exp \left(-c \gamma t_{12}\right),
$$


where $\gamma$ is the dephasing rate and the constant $c$ takes the value 2 (4) in case of homogeneous (inhomogeneous) broadening. In this two-level approach, the dephasing time $T_{2}=1 / \gamma$, reflecting the homogeneous linewidth of the resonance, can be obtained from the experiment.

For the transient light-induced grating (TLIG) experiments, the delay between the two first (pump) laser pulses $t_{12}$ is set to zero. The interference pattern creates a real population grating in the material. The period of the grating is $\Lambda=\lambda /(2 \sin (\theta / 2))$, where $\lambda$ is the pulse wavelength and $\theta$ is the angle between the wave vectors $\mathbf{k}_{1}$ and $\mathbf{k}_{2}$. The third (probe) pulse with wave vector $\mathbf{k}_{3}$ arrives at the sample delayed by time $t_{23}$ and is diffracted by the induced grating into the directions $\mathbf{k}_{3} \pm\left(\mathbf{k}_{2}-\mathbf{k}_{1}\right)$. The modulation of the exciton density $\Delta N$ in the grating decays due to recombination and diffusion. Assuming an exciton mean-free path much smaller than the grating period, the decay is described by the rate equation

$$
\frac{\partial \Delta N}{\partial t}=D \nabla(\Delta N)-\frac{\Delta N}{T_{1}}
$$

where $D$ is the diffusion constant and $T_{1}$ is the exciton recombination lifetime. The intensity of the first diffracted order decreases exponentially $I_{\text {TLIG }} \propto \exp \left(-2 t_{23} / T_{D}\right)$. The grating decay time $T_{D}$ for the sinusoidal grating is given by

$$
\frac{1}{T_{D}}=\frac{1}{T_{1}}+\frac{4 \pi^{2} D}{\Lambda^{2}} .
$$

By measuring $T_{D}$ as a function of $1 / \Lambda^{2}$, one can determine the diffusion constant $D$. Extrapolation to $\Lambda=\infty$ (no spatial modulation) gives the exciton lifetime $T_{1}{ }^{11}$

In both types of the experiments, the grating vector $\mathbf{k}_{2}-\mathbf{k}_{1}$ is set parallel to the layers in GaSe. The pump and probe pulses in the dephasing experiments are polarized perpendicular to their plane of incidence and their intensity ratio is $2: 1$. In the transient grating experiment the same intensity ratio and polarization is maintained between the two pump pulses. To minimize the coherent interaction between the pump and probe pulses for short delays $t_{23}$, the probe pulse is ten times weaker and crosspolarized with respect to the pump pulses. The laser beams are focused on the sample to a spot size of $50 \mu \mathrm{m}$. The signal is detected, time-integrated, by a spectrometer which has a resolution of $0.1 \mathrm{meV}$ and recorded by an optical multichannel analyzer (OMA) system. Low temperatures between 7.5 and $65 \mathrm{~K}$ are maintained in a liquidhelium bath cryostate.

In general, the center and width of the exciton line, as well as its fine structure, depend slightly on the position of the illuminated spot. We were very cautious to maintain the same spot position during the measurements. This was accomplished by a CCD-image system imaging an area of $300 \times 200 \mu \mathrm{m}^{2}$ of the sample on a monitor live during the experiments.

\section{EXPERIMENTAL RESULTS AND DISCUSSION}

\section{A. Dephasing experiment}

In the dephasing experiments only the excitons, populating their initial coherent states, contribute to the DFWM signal. The exciton population usually persists long after the phase coherence is lost, and undergoes energy relaxation toward an equilibrium distribution. It is useful to compare the spectral features of the optical nonlinearity, observed in the dephasing and transient grating experiments, with the linear absorption of the sample (Fig. 1). The maximum of the coherent signal is shifted to the high-energy side of the linear absorption line. Shortly after the excitation $\left(t_{23}<10 \mathrm{ps}\right)$, the signal in the transient grating experiment coincides spectrally with the dephasing signal. However, as indicated in Fig. 1, already at $t_{23}=35 \mathrm{ps}$ after the excitation, its spectral peak occurs closer to the center of the linear absorption line, approximately $0.3 \mathrm{meV}$ below the peak in the coherent signal. The fine structure in the linear absorption is partially present in the nonlinear spectra as well.

The spectral shape of the coherent signal is sensitive to the excitation density, as shown in Figs. 2(a) and 2(b) with normalized DFWM spectra at different excitation levels and two different delay times. An increase in the excitation density shifts substantially the main peak in the spectra to higher energies and narrows it. These trends are more pronounced at $t_{12}=0$, when the two incident pulses are simultaneous. Along with the main peak at $2.1095 \mathrm{eV}$, smaller maxima at 2.108 and 2.1106 $\mathrm{eV}$ can be seen in Fig. 2. Their position and relative amplitudes are somewhat more accidental than that of the main peak and, in the following, we will mainly analyze the spectra around the main peak in the signal. For both delays, the fine structure gradually disappears with an increase in intensity.

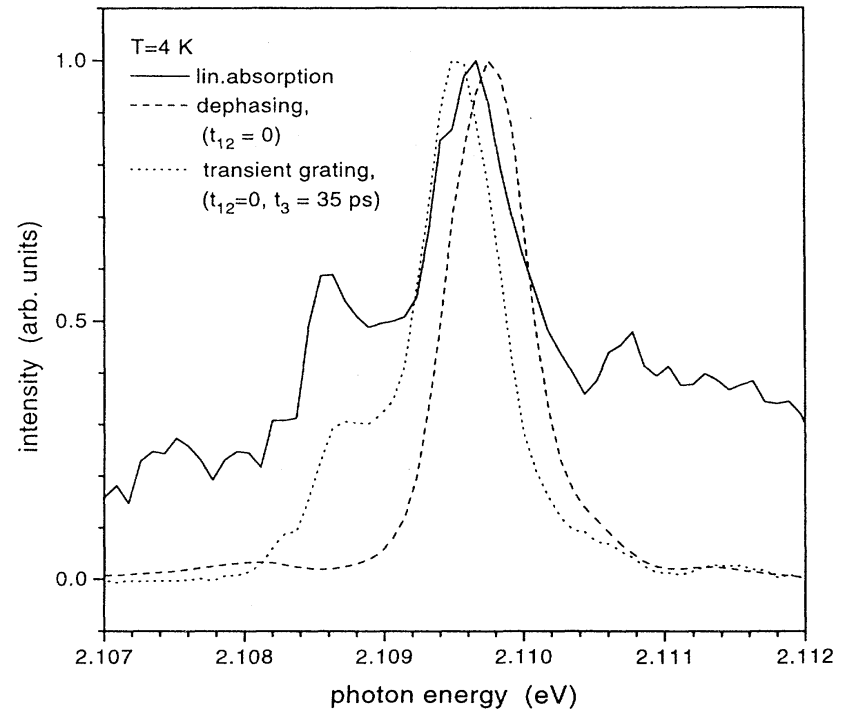

FIG. 1. The signal spectra in dephasing and transient grating experiments, and the linear transmission of the sample. 


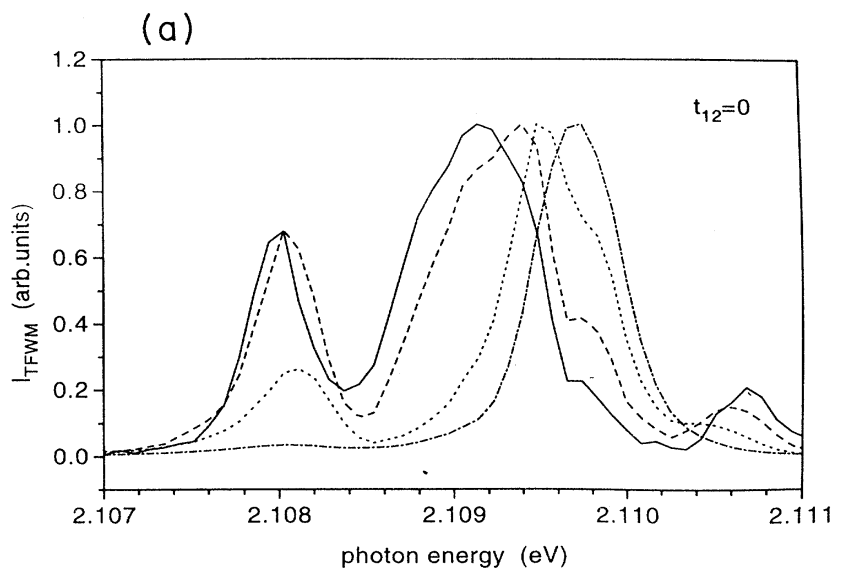

(b)

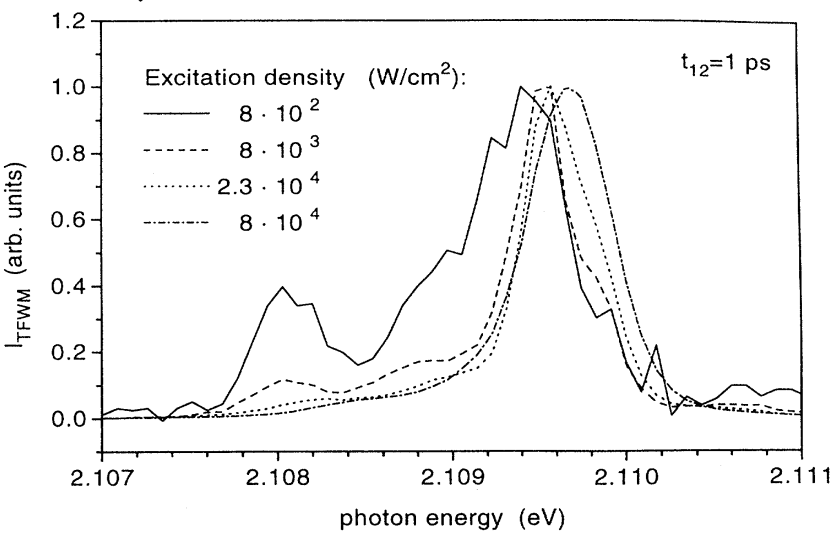

FIG. 2. Normalized spectra of the coherent signal for different excitation intensities at $t_{12}=0$ (a) and $t_{12}=1 \mathrm{ps} \mathrm{(b).}$

Assuming a level saturation as the only mechanism of the nonlinearity, it is difficult to explain the observed shifts, since saturation in a two-level system reduces the oscillator strength, which by itself does not modify the line shape or center frequency of the nonlinear signal. To obtain insight into the nonlinear mechanism in GaSe, we examine below the dephasing process, spectrally and temporally, at two excitation levels, different by one order of magnitude. The situation at an excitation intensity of $8 \times 10^{3} \mathrm{~W} / \mathrm{cm}^{2}$ is shown in Fig. 3. Regarding the signal evolution in time [see Figs. 3(a) and 3(c)], two distinct parts can be seen: a spike around $t_{12}=0$, i.e., an instantaneous part coincident with the overlap in time between the pump and probe pulses, and a rise and subsequent decay, asymmetric with respect to $t_{12}=0$; the delayed peak is a characteristic feature of the time integrated photonecho signal in case of strong inhomogeneous broadening. ${ }^{12}$ In this case, the homogeneous linewidth of 0.1 $\mathrm{meV}$ is estimated from the fit of the form in Eq. (1), assuming $c=4$.

The relative ratio between the instantaneous contribution and the photon-echo contribution is strong spectrally dependent. As seen from Figs. 3(a) and 3(b) the photon-echo-like features are strong only in the higher-

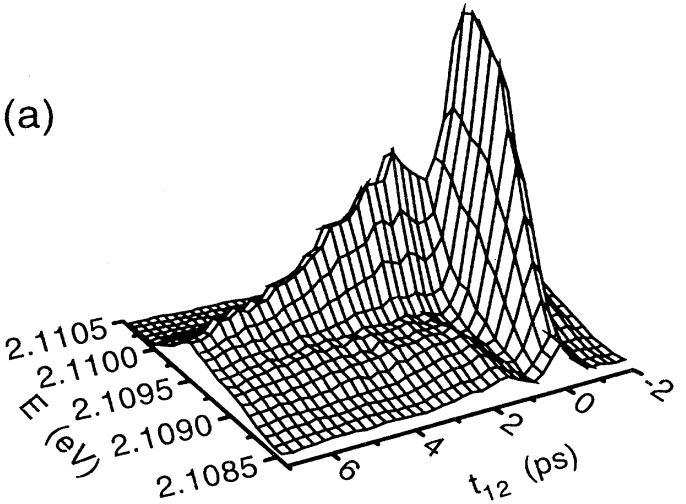

(b)
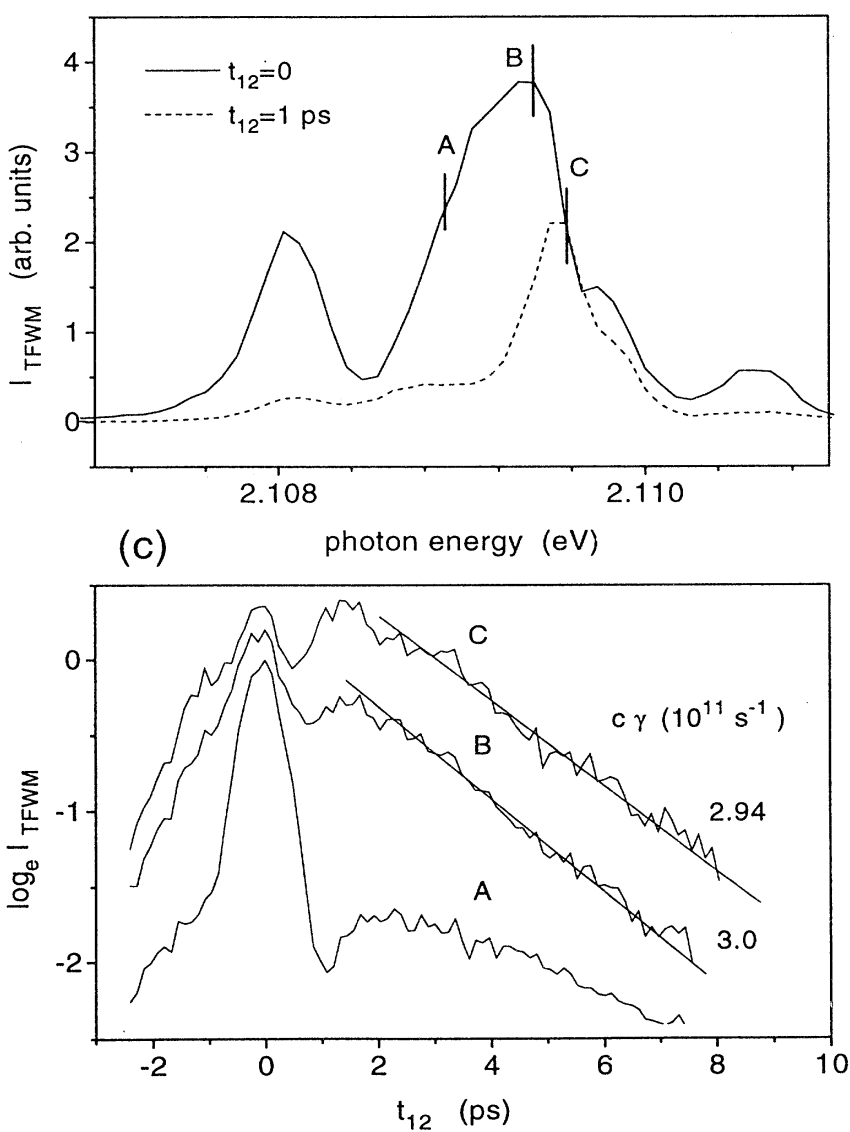

FIG. 3. The spectral and temporal dynamics of the coherent signal. Three-dimensional plot with linear scale of the $z$ axis (a), spectra at $t_{12}=0$ and $t_{12}=1 \mathrm{ps}(\mathrm{b})$, and normalized temporal profiles (offset for clarity) at selected spectral positions (c). The straight solid lines are exponential fits of the form in Eq. (1) with the values of $c \gamma$ given besides. The photon energies, corresponding to the labels are $E_{A}=2.1089 \mathrm{eV}, E_{B}=2.1094 \mathrm{eV}$, and $E_{C}=2.10957 \mathrm{eV}$; the excitation density is $8 \times 10^{3} \mathrm{~W} / \mathrm{cm}^{2}$.

energy part of the signal around $C$, whereas in the lowerenergy part close to $A$ and $B$ the overlap signal dominates. Within the narrow spectral range where the photon-echo features are strong, the signal decay rate is nearly independent of the spectral position.

The distinction between the instantaneous signal and 
the photon-echo signal can also be regarded as a temporal quenching of the DFWM signal for a certain delay, due to complete saturation of the corresponding (localized) states, as will be discussed in the following. For low intensity (Fig. 3), this saturation needs both pulses and occurs for a small but final delay. For higher excitation density, as in Fig. 4, complete saturation occurs by one pulse alone, and the quenching of the DFWM original occurs already at $t_{12}=0$.

The photon-echo component increases with the excitation much faster than the instantaneous component. An excitation density of $8 \times 10^{4} \mathrm{~W} / \mathrm{cm}^{2}$ is enough to make the photon-echo behavior dominant over the whole spectral range (Fig. 4). The decay rate increases with excitation and becomes spectrally dependent, as can be seen from the values of $c \gamma$, given in Fig. 4(c). The signal peaks closer to $t_{12}=0$ on the high-energy side of the signal, around $G$ and $H$ in Fig. 4(c), while on the low-energy side $\left(D, E\right.$, and $F$ ) the maximum is delayed by $t_{12} \approx 2 \mathrm{ps}$.

To account for these observations, we refer to the specific structure of GaSe along the crystallographic $c$ axis. In this direction, stacking fault disorder creates a band, described by a one-dimensional (1D) disordered chain Hamiltonian and density-of-states function. The process of the exciton localization along the $c$ axis corresponds to the energy relaxation within the band. The relaxation probabilities differ somewhat in the lower and upper parts of the band. Studies of luminescence under cw excitation ${ }^{13}$ show that excitons created below the center of the emission line predominantly retain the same energy during their lifetime, while excitons created above the line center localize by relaxing into deeper states before the recombination. The explanation of our findings is based on the assumption that the localization occurs on a time scale of a few picoseconds after the excitation, i.e., comparable with the pulse duration in our experiments. Pulsed laser excitation polarized in the plane $\mathbf{E} \perp \mathbf{c}$ will initially populate the entire disordered band around $\mathbf{k}_{\perp c}=\mathbf{0}$, provided that the pulse spectrum is broader than the exciton absorption line. The values of the exciton interband relaxation times given in the literature ${ }^{7-9}$ and in this work (see transient grating results) vary in the broad range between $80 \mathrm{ps}$ and $1.3 \mathrm{~ns}$. Even for the shortest estimates, the ratio between the intraband and interband relaxation times is small, and the localization rapidly fills the states below the line center. By the time the pump pulse leaves the sample, a significant part of the localized states is completely saturated as a consequence of the direct excitation and subsequent relaxation. The probe pulse, arriving with positive delay, does not feel any nonlinearity at the corresponding energies. The excitons, initially created in the localized states, thus cannot contribute to the coherent signal even while they stay coherent. The grating is created only at higher energies, corresponding to the extended states,and the signal can be seen there.

This description roughly matches the situation shown in Fig. 3, where there is a big difference between the strength of the photon-echo signal in the low- and highenergy parts of the DFWM spectrum. The pump-probe overlap signal, instant with respect to $t_{12}=0$, is intense at

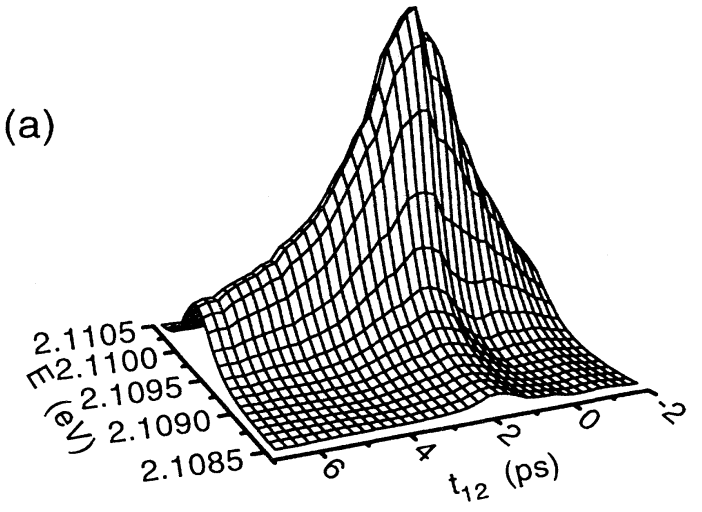

(b)
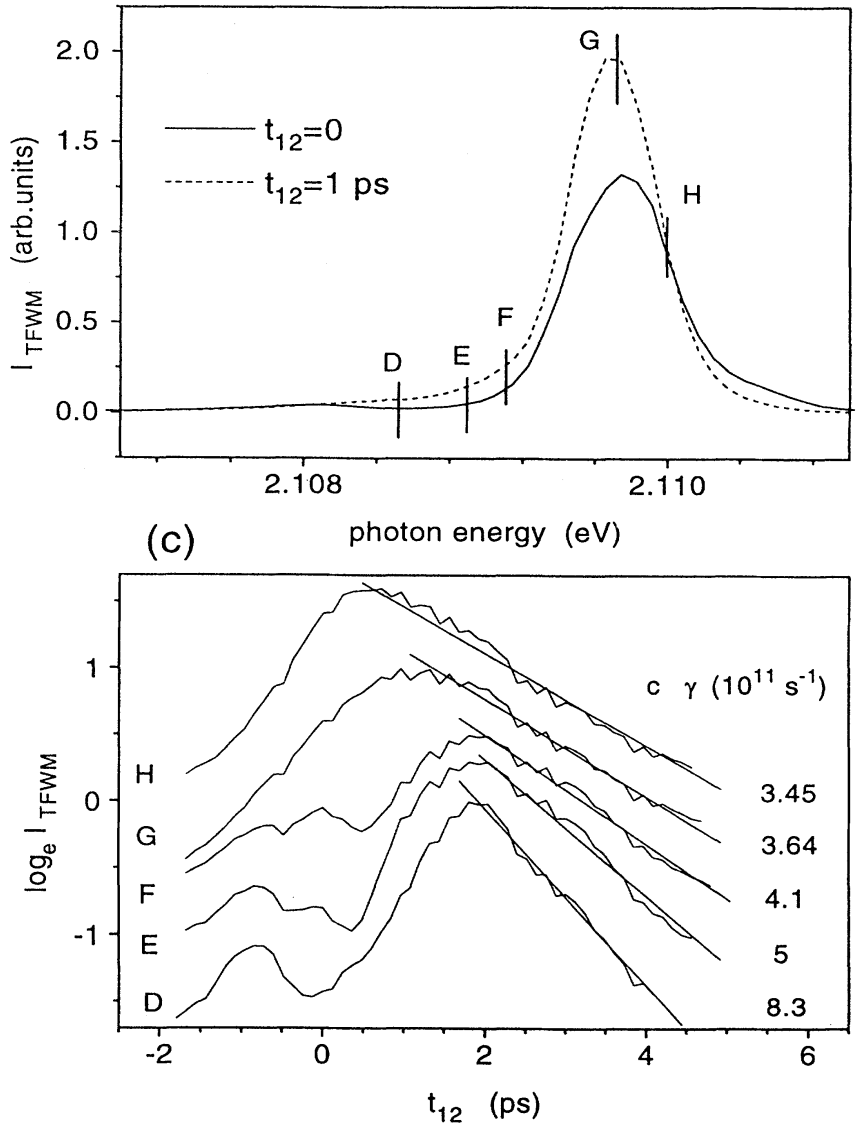

FIG. 4. The same dependences as in Fig. 3, with the excitation density $8 \times 10^{4} \mathrm{~W} / \mathrm{cm}^{2}$. The photon energies corresponding to the labels are $E_{D}=2.10854 \mathrm{eV}, E_{E}=2.1089 \mathrm{eV}, E_{F}=2.1091$ $\mathrm{eV}, E_{G}=2.1097 \mathrm{eV}$, and $E_{H}=2.1099 \mathrm{eV}$.

lower energies, manifesting the initial existence of the grating. The relative weakness of the photon-echo signal, which in the real time is delayed by $2 t_{12}$, indicates that the relaxation has already saturated the localized states by this time.

The energy relaxation leading to the exciton localization infers a change of the exciton center-of-mass coordi- 
nate. Regarding its magnitude, we assume the relaxation into the nearest-neighboring potential wells is mainly responsible for the observed complete saturation of the localized states. In the direction of the $c$ axis, the stacking fault disorder influences the excitonic states over the length scale of $5-10 \mathrm{GaSe}$ layers $(40-80 \AA),{ }^{13}$ i.e., comparable with the exciton Bohr radius of $32 \AA$. A similar characteristic length can be ascribed to the change in the exciton center-of-mass position along the $c$ axis during the energy relaxation. On the longer length scale, the macroscopic regions in the sample, having slightly different parameters of the exciton band, like the center energy and width, do not exchange energy in the process of localization. The picture of localization and saturation, presented above, can be applied to such regions separately, as for the different samples. In the experiment we see the integrated response from all regions, including those where the center or bottom of the exciton band is below the average values for the entire sample. As a result, some states are not saturated by the localization, even if their optical response is seen in the lower-energy part of the linear absorption and DFWM spectra. The low density of such states makes their contribution to the signal small, compared with a much stronger signal from the regions with average parameters of the excitonic band. For the same reason they may become temporarily saturated by the excitation alone. This is indeed seen at higher intensities around energies $D$ and $E$ in Fig. 4, as a dip at $t_{12}=0$, in contrast to Fig. 3 , where the overlap results in a peak and the saturation occurs for a small delay. The temporal widths of the peak and the dip are approximately the same, indicating that the saturation lasts only as long as the pump pulse is present. This saturation is also reflected in Fig. 2(a) as a blue shift, and narrowing from the low-energy side of the DFWM signal which becomes more pronounced for $t_{12}=0$.

The spectral dependences of the decay shown in Fig. 4(c) can be treated according to the recent studies of spectrally resolved transient DFWM. ${ }^{12}$ The characteristic features of the time-integrated photon echo gradually disappear, and the decay rate $\gamma$ increases at higher energies, indicating an increased ratio between the homogeneous and inhomogeneous linewidths. The fundamental source of the increased homogeneous broadening is a decrease in state lifetime for the upper part of the band due to the fast localization. We note that, in the previous studies, ${ }^{8}$ a strong relation between the spectral relaxation and dephasing was suggested. An additional reason for the resonance homogenization is that at higher excitation densities the signal shifts to higher energy, where the contribution from extended excitonic states to the nonlinear signal is stronger.

The optical nonlinear mechanism in GaSe thus is dominated by the features that are characteristic of the bandfilling nonlinearity. The most common feature of the independent carrier band filling is a blueshift of the absorption edge with increased excitation. In $\mathrm{GaSe}$ it is observed for the excitons, which are, to the first approach, bosons. However, the origin of the excitonic blueshift is similar to the case of the independent carriers. The fermionic nature of bound electron-hole pairs becomes im- portant in the localized states, making them eventually unavailable for the optical population.

\section{B. Transient grating experiments}

By spectrally resolving the DFWM signal in incoherent transient grating experiments, we obtain signal decays that depend on the grating constant as well as the energy.

Typical spectra of the signal at $10 \mathrm{~K}$ are shown together with the linear absorption of the sample in Fig. 5(a). The excitation density is $8 \times 10^{4} \mathrm{~W} / \mathrm{cm}^{2}$, and the grating period $\Lambda=11.7 \mu \mathrm{m}$. Shortly after the excitation $\left(t_{23}=10\right.$ ps), the signal is blueshifted by approximately $0.3 \mathrm{meV}$ with respect to the center of the linear absorption line. The peak in the spectra at larger delays (spectra at 150 and $1000 \mathrm{ps}$ ) is close to the maximum of the linear absorption. Comparatively little change in the spectral shape of the DFWM signal occurs between 500 and 1400 ps, except for an exponential decrease of all spectral components.

(a)

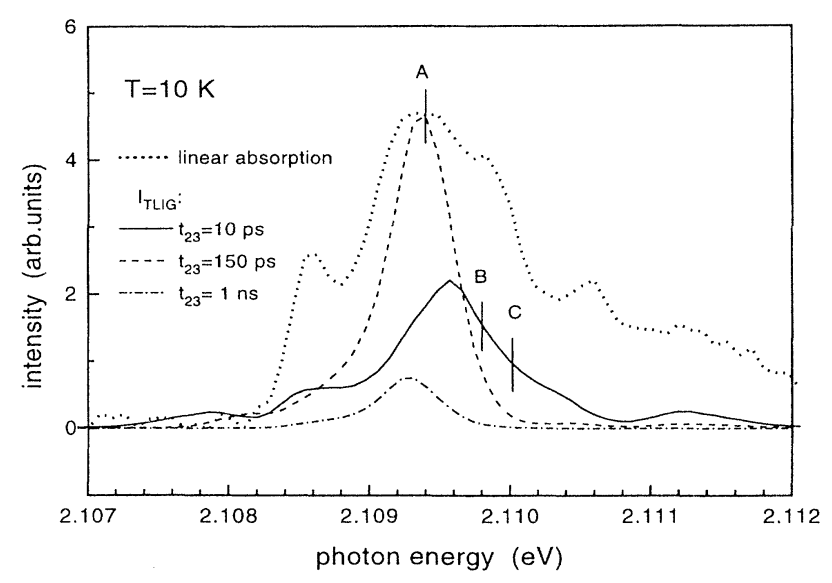

(b)

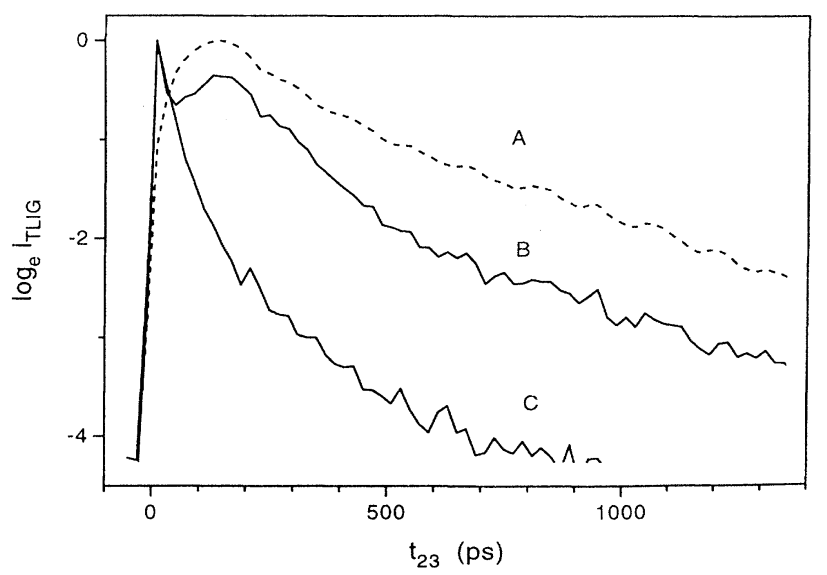

FIG. 5. The spectral and temporal dynamics of the signal in the transient grating experiment. Spectra at $t_{23}=10 \mathrm{ps,} 150 \mathrm{ps}$, and $1 \mathrm{~ns}$ (a); normalized temporal profiles at selected spectral positions (b). $E_{A}=2.1094 \mathrm{eV}, E_{B}=2.1098 \mathrm{eV}$, and $E_{C}=2110$ $\mathrm{eV}$. The excitation density $8 \times 10^{4} \mathrm{~W} / \mathrm{cm}^{2}$. 
Figure 5(b) shows the time evolution of the signal at the spectral positions labeled $A, B$, and $C$. At lower energies around $A$, it continues to rise for $0<t_{23}<150 \mathrm{ps,}$ while the signal at higher energies around $C$ rapidly decreases with the decay time of about 100 ps. At the intermediate position $B$ both types of behavior are seen. The slow exponential decay at $A$ and $B$ with the decay time $T_{D}=1200 \mathrm{ps}$ is characteristic for $t_{23}>500 \mathrm{ps}$, and the dependence of $T_{D}$ on the grating period is in agreement with relations (2) and (3).

The explanation of this behavior is based on the results of the dephasing experiment. The excitons, created in the higher-energy part of the band, localize, saturating its lower part. Shortly after the excitation the DFWM signal is shifted to higher energies, as in the dephasing experiment.

For longer delays $t_{23}$, interband relaxation will become effective. It will depopulate the excitonic band, removing the saturation from its lower part. This will allow for the grating that previously existed only at higher energies to relax to the again available localized states. The localization is thus responsible for the rapid signal decay on the high-energy side of the signal spectrum and its increase on the low-energy side, provided that the spatial diffusion rate is much smaller than the recombination rate. Such behavior is seen in Fig. 5, where the energies labeled $A$ and $C$ can be regarded as lower and higher, respectively.

It is impossible to account for all the influences to the signal due to intraband relaxation, recombination, and diffusion at each spectral component. The initial parts of the decay indicate the absence of the equilibrium between these processes. Nevertheless, after $t_{23} \approx 500$ ps the uniform exponential decay at all frequencies indicates steady conditions, and the diffusion model can be applied to characterize the exciton transport.

The spectral parts of the signal, characterized by different temporal behaviors, also have different dependences on the excitation density. The slowly decaying part, detected at lower energies, only weakly depends on the pump intensity. It indicates that the initial exciton population eventually has little influence on the amplitude of the grating at lower energies. In contrast, the rapidly decaying part in the high-energy side of the signal strongly increases with excitation. At higher pump intensities it may substantially exceed the slowly decaying part, which then can be mistaken as a weak, timeindependent background. This can explain the difference between our findings and the results of previous TLIG experiments ${ }^{7}$ obtained at higher excitation intensities, where only the rapid initial part $\left(T_{D} \approx 80\right.$ ps at $\left.15 \mathrm{~K}\right)$, solely attributed to recombination, was observed. We find the decay with similar time only on the high-energy side of the signal around the position $C$ in Fig. 5. This decay most likely corresponds to a variety of processes including localization, recombination, scattering to the indirect valley, ${ }^{8}$ bound exciton formation, ${ }^{10}$ and the spatial washout of the grating.

The spectral positions $A$ and $C$ and the excitation intensity in Fig. 5 are chosen so as to better illustrate the pure types of characteristic behavior. In fact, both types overlap in spectral and time domain (as at $B$ ) and the overlap increases with intensity. In further studies we maintain the same excitation intensity $8 \times 10^{4} \mathrm{~W} / \mathrm{cm}^{2}$ that provides a reasonable compromise between a negligible influence of the initial part at $t_{23}>500$ ps and a fairly good signal-to-noise ratio.

As was already mentioned, the fine structure observed in the linear and nonlinear spectra is position dependent. It shows that the statistical properties of the stacking fault disorder are nonuniform even in directions parallel to the GaSe layers. If a nonuniformity of such kind is present on length scales shorter than the exciton diffusion length, it could also contribute to the exciton scattering or localization. Therefore, it would be interesting to analyze the dependence of the diffusion constant on the exciton energy to see the influence of disorder in these directions. However, the latter dependence was below the resolution of our system, limited by fluctuations in the laser wavelength within one measurement cycle. Under these circumstances, we deduce the diffusion constant as a function of temperature from the signal, spectrally integrated around the main peak.

The decay of such a signal at $10 \mathrm{~K}$ for various grating constants and the determination of $D$ and $T_{1}$ are shown in Figs. 6(a) and 6(b). The reproducibility of the results is

(a)

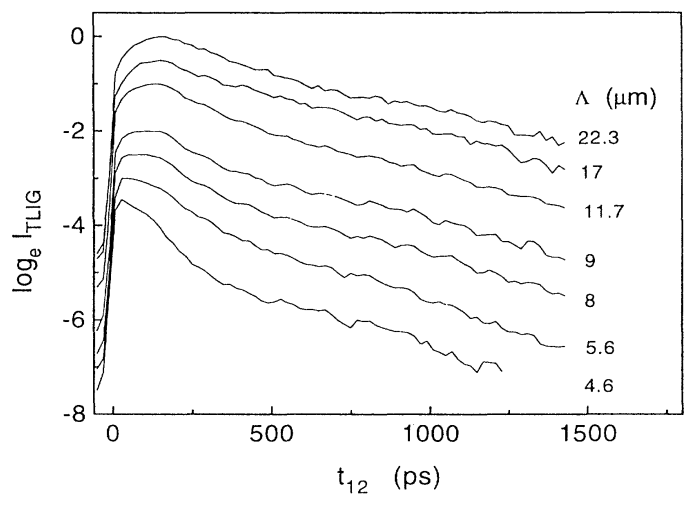

(b)

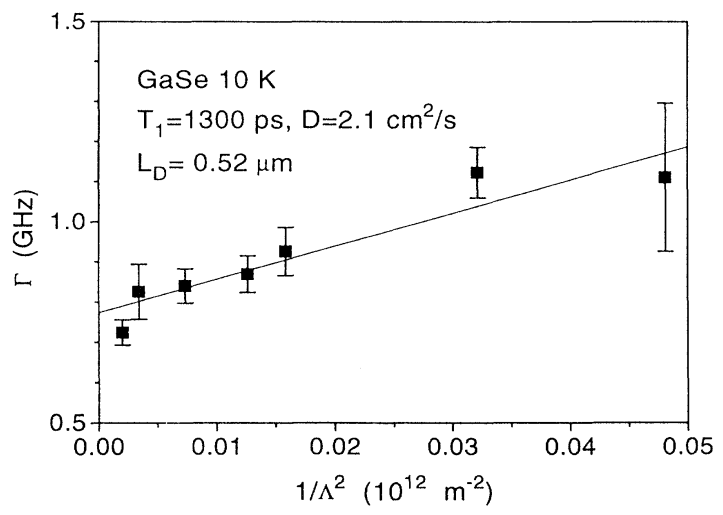

FIG. 6. (a) The decay of the spectrally integrated signal for different grating constants $\Lambda$; the curves are offset for clarity. (b) The determination of the diffusion coefficient and the lifetime. 


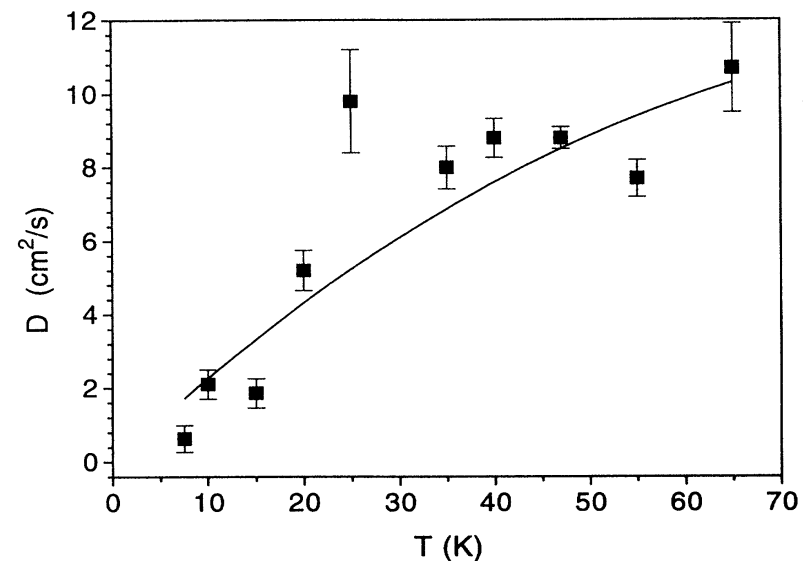

FIG. 7. The temperature dependence of the diffusion constant $D$ (squares) with the fit (solid line) from Eq. (4), assuming $1 / \tau_{p}=\alpha T^{3 / 2}+\Gamma_{0}$, where $\Gamma_{0}=1.48 \times 10^{12} \mathrm{~s}^{-1}$ and $\alpha=1.32 \times 10^{9}$ $\mathbf{s}^{-1} \mathbf{K}^{-3 / 2}$.

within the error bars. Obtained temperature dependences of $D$ and $T_{1}$ are plotted in Figs. 7 and 8 .

The diffusion coefficient is related to the momentum relaxation time $\tau_{p}$ as

$$
D=\frac{k_{B} T}{m^{*}} \tau_{p} \text { for } t \gg \tau_{p},
$$

where $m^{*}=m_{e}+m_{h}$ is the exciton effective mass, $m_{e}$ and $m_{h}$ are effective electron and hole masses, $k_{B}$ is the Boltzmann constant, and $T$ is the temperature of the exciton gas. If various scattering mechanisms contribute to the momentum relaxation, we assume that $\tau_{p}$ can be described via the sum of scattering rates $\Gamma_{i}$ :

$$
\frac{1}{\tau_{p}}=\sum_{i} \Gamma_{i}
$$

where index $i$ denotes different scattering mechanisms.

Measurements of free-carrier Hall mobility in GaSe above $77 \mathrm{~K}$ (Ref. 6) show a predominant influence of

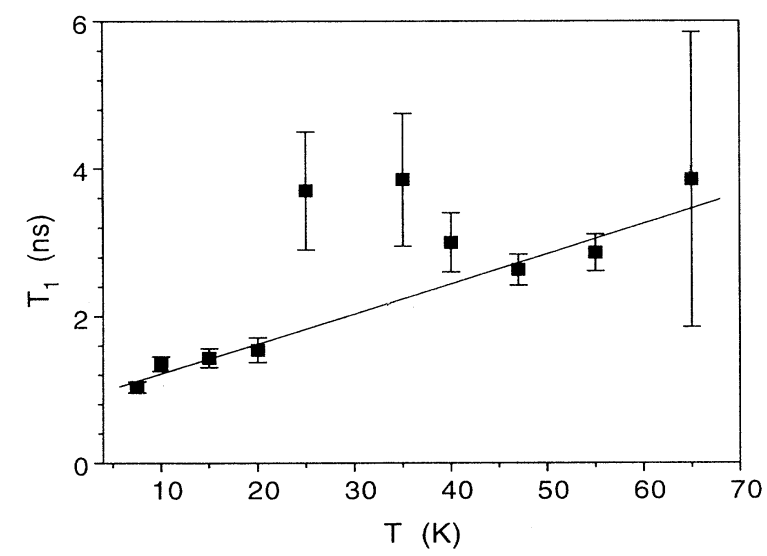

FIG. 8. The temperature dependence of the exciton recombination lifetime $T_{1}$. The straight solid line is a linear fit of the form $T_{1}=0.806+0.04 T(\mathrm{~ns})$. optical-phonon scattering. The lowest mode, that is believed to be strongly coupled to carriers, has an energy of $17 \mathrm{meV}$. At low temperatures the optical-phonon population is small and can be neglected, but scattering by acoustic phonons is important. The momentum relaxation rate for exciton-acoustic-phonon scattering via deformation potential scattering depends on the temperature as ${ }^{14}$

$$
\Gamma_{\mathrm{ad}} \propto\left(m^{*} T\right)^{3 / 2} .
$$

Exciton-acoustic-phonon scattering via the piezoelectric potential is strongest parallel to the crystallographic $c$ axis, and since we study the diffusion perpendicular to $c$ we take into account only deformation potential scattering. Figure 7 plots experimentally determined values of D. A simplified treatment of this dependence is given in the same figure, by introducing a temperatureindependent scattering (trapping) rate $\Gamma_{0}$. The solid line is a fit of the form in Eq. (4), assuming $1 / \tau_{p}=\alpha T^{3 / 2}+\Gamma_{0}$, where $\alpha$ and $\Gamma_{0}$ are adjustable parameters. If only $\Gamma_{\mathrm{ad}}$ is accounted for, $D \propto T^{-1 / 2}$ as $T$ approaches zero, and a big discrepancy with experimental data arises at low temperatures. A substantial scattering (trapping) rate $\Gamma_{0}=1.48 \times 10^{12} \mathrm{~s}^{-1}$ thus accounts for the decrease in $D$ at low temperatures. From the relation in Eq. (4), assuming thermal equilibrium and exciton effective mass $m^{*}=0.44 m_{0}$ (Ref. 15) ( $m_{0}$ is the free-electron mass), the momentum relaxation time $\tau_{p}=0.6 \mathrm{ps}$ at $10 \mathrm{~K}$ is deduced. Clearly, by evaluating the diffusion constant for $t_{23}>500 \mathrm{ps}$ we have fulfilled the requirement $t \gg \tau_{p}$ in (4). It is interesting to compare the value obtained with the average dephasing time $T_{2} \approx 6$ ps at $4 \mathrm{~K}$ and the same excitation density. The difference is too large to be explained by the different lattice temperature. A similar difference is also found in GaAs MQW structures, ${ }^{5}$ and the explanation presented herein is plausible for GaSe as well: the optically created excitons have to acquire momentum before they can feel other mechanisms of scattering than acoustic phonons. But then they lose phase coherence and cease to contribute to the photonecho signal. Hence the coherent population is scattered only by acoustic phonons.

The temperature dependence of the exciton lifetime $T_{1}$ is shown in Fig. 8. The solid line with the slope $40 \mathrm{ps} / \mathrm{K}$ is a linear fit to the obtained dependence. The rather sharp increase in $T_{1}$ between 20 and $25 \mathrm{~K}$ may be an artifact. A similar increase in $D$ between 15 and $25 \mathrm{~K}$ can be seen in Fig. 7. The two quantities are related together via Eq. (3), and if any experimental error is present in $D$ (slope), it will show up even more strongly in $T_{1}$. At present, we do not have any explanation for a simultaneous deviation in $T_{1}$ and $D$ from the simple relations expressed by the solid lines in Figs. 7 and 8 .

The lifetime increases slightly with temperature due to an increased occupation of nonradiative states $^{16}$ and a thermal emptying of deep and impurity-bound states that enhance radiative and nonradiative recombinations. ${ }^{17}$ In our experiments, we cannot distinguish between two contributions to the lifetime, but recent time-resolved luminescence measurements ${ }^{9}$ show that the radiative 
TABLE I. Diffusion constant and lifetime in GaAs and other chalcogenides.

\begin{tabular}{lcccc}
\hline \hline & $\mathrm{CdS}_{1-x} \mathrm{Se}_{x}, 20 \mathrm{~K}$ & $\mathrm{GaSe}, 10 \mathrm{~K}$ & $\mathrm{CdS}, 7.4 \mathrm{~K}$ & $\mathrm{CdSe}, 10 \mathrm{~K}$ \\
\hline$D, \mathrm{~cm} / \mathrm{s}^{2}$ & 0.1 & 2.1 & 13.0 & $7.0^{\mathrm{a}}, 50.0^{\mathrm{b}}$ \\
$T_{1}, \mathrm{~ns}$ & 2.66 & 1.3 & 1.0 & 2.0 \\
\hline \hline
\end{tabular}

${ }^{a}$ In direction $\perp c$.

${ }^{\mathrm{b}}$ In direction $\| c$.

recombination rate decreases at higher temperatures, thus causing the observed increase in the lifetime. Values of $T_{1}$ obtained from luminescence decay are similar to our transient grating results.

The temperature range in the transient grating experiments was limited to above $7.5 \mathrm{~K}$, because at lower temperatures the dependence of the decay time on the grating period becomes irreproducible. Supposedly, excitons are localized by impurities and stacking fault inhomogeneities even in the direction parallel to the layers, and the diffusion model is invalid at the lowest temperatures. The temperature is limited upwards due to low signal at higher temperatures. An increase in temperature gradually removes the fine structure and broadens the DFWM signal. At $65 \mathrm{~K}$, the spectrum has no fine structure and is about three times broader than at $10 \mathrm{~K}$.

Now we can compare the properties of GaSe and other chalcogenides investigated previously. ${ }^{2-4,18}$ This is done in Table I. Concerning the transport properties at low temperatures, GaSe occupies an intermediate position between crystalline materials and disordered $\mathrm{CdS}_{1-x} \mathrm{Se}_{x}$ mixed crystals. The exciton lifetime in GaSe is quite typical for that class of materials. Having in mind the quasi-two-dimensional (2D) character of the exciton transport, one can also compare parameters of GaSe and other quasi-2D system, namely $100-\AA$ GaAs multiple quantum wells, characterized by $T_{1}=0.6 \mathrm{~ns}$ and $D=20$ $\mathrm{cm} / \mathrm{s}^{2}$ at $10 \mathrm{~K} .{ }^{5}$

Thus the low-temperature results show that exciton transport along GaSe layers is significantly affected by the disorder, present over a length scale shorter than the exciton diffusion length $(\approx 1 \mu \mathrm{m})$.

\section{CONCLUSIONS}

In our dephasing and transient light-induced grating experiments, we detect the important influence of band filling on the excitonic optical nonlinearities in GaSe. The band filling is a consequence of the fast exciton localization that occurs in directions parallel to the crystallographic $c$ axis. Although with the picosecond pulses we could not follow the temporal evolution of the band filling, we detected its implications on a picosecond time scale, taking advantage of our spectral resolution.

In the transient light-induced grating experiment, we found two types of dynamic behaviors in the diffracted signal. The fast exponential decay with $T_{D} \approx 100 \mathrm{ps}$ was found on the high-energy side of the spectrum, in compliance with previous TLIG studies. ${ }^{8,9}$ This decay is ascribed to a variety of relaxation processes, rather than to exciton recombination alone. On the low-energy side of the signal, an initial increase and subsequent slow exponential decay, extending to nanosecond times, was found. We use it to evaluate the exciton transport properties. The estimated nanosecond exciton lifetimes are close to those obtained using the time-resolved luminescence and transmission techniques. ${ }^{7,10}$ We treat the obtained temperature dependence of the diffusion constant qualitatively by introducing a large temperatureindependent scattering (trapping) rate. This illustrates that a substantial disorder is present in GaSe even in the directions of the crystalline layers.

\section{ACKNOWLEDGMENTS}

The authors would like to thank K.-H. Pantke, I. Balslev, and D. Birkedal for many helpful discussions. This work was supported by the Danish Natural Science Research Council and the Danish Research Academy. Part of it was performed at Fysisk Institut, Odense University.
"Permanent address: Dept. of Solid State Electronics, Vilnius University, Sauletekio Ave. 9, Bldg. III, 2054 Vilnius, Lithuania.

$\lceil$ Permanent address: Institute of Microelectronics Technology and Superpure Materials, Chernogolovka, Moscow District 142432, Russia.

$\ddagger$ Permanent address: Fysisk Institut, Odense Universitet, Campusvej 55, DK-5230 Odense M, Denmark.

${ }^{1} \mathrm{~J}$. Forney, K. Maschke, and E. Mooser, J. Phys. C 10, 1887 (1977).

${ }^{2}$ C. Weber, U. Becker, R. Renner, and C. Klingshirn, Z. Phys. B
72, 379 (1988).

${ }^{3}$ J. Erland et al., Phys. Rev. B 47, 3582 (1993).

${ }^{4}$ H. Schwab, K.-H. Pantke, J. M. Hvam, and C. Klingshirn, Phys. Rev. B 46, 7528 (1992).

${ }^{5}$ D. Oberhauser et al., Phys. Rev. B 47, 6827 (1993).

${ }^{6}$ R. Fivaz and P. Schmid, in Optical and Electrical Properties, Vol. 4 of Physics and Chemistry of Materials with Layered Structures, edited by P. Lee (Reidel, Dordrecht, 1976), pp. $343-384$.

${ }^{7}$ A. Hasegawa et al., J. Lumin. 53, 371 (1992).

${ }^{8}$ J. M. Hvam and C. Dörnfeld, in Optical Switching in Low- 
Dimensional Systems, edited by L. Bányai and H. Haug (Plenum, New York, 1989), pp. 233-241.

${ }^{9}$ F. Minami, A. Hasegawa, S. Asaka, and O. Inoue, J. Lumin. 45, 409 (1990).

${ }^{10}$ X.-C. Zhang, M. Gal, and A. Nurmikko, Phys. Rev. B 30, 6214 (1984).

${ }^{11}$ H. Eichler, P. Günter, and D. Pohl, Laser-Induced Dynamic Gratings, Springer Series in Optical Sciences Vol. 50 (Springer-Verlag, Berlin, 1986), p. 256.

${ }^{12}$ J. Erland et al., Phys. Rev. B 50, 15047 (1994).
${ }^{13}$ V. Capozzi and C. Maschke, Phys. Rev. B 34, 3924 (1986).

${ }^{14}$ N. Zinov'ev et al., Zh. Eksp. Teor. Fiz. 84, 2153 (1983) [Sov. Phys. JETP 57, 1254 (1983)].

${ }^{15}$ V. Capozzi, L. Pavesi, and J. Staehli, Phys. Rev. B 47, 6340 (1993).

${ }^{16}$ L. Andreani, F. Tassone, and F. Bassani, Solid State Commun. 77, 641 (1991).

${ }^{17}$ D. Oberhauser et al., Phys. Status Solidi B 173, 53 (1992).

${ }^{18}$ H. Schwab et al., Phys. Status Solidi B 172, 479 (1992). 